\title{
MUON RING COOLER FOR THE MUCOOL EXPERIMENT*
}

\author{
V. Balbekov ${ }^{\dagger}$, S. Geer, N. Mokhov, R.Raja, Z.Usubov, FNAL, Batavia, IL 60510, USA
}

\begin{abstract}
A possibility to use a ring cooler for the MUCOOL experiment is considered. The cooler is a ring accelerator of about $37 \mathrm{~m}$ in circumference consisting of 8 dipole magnets, 4 short solenoids with a field flip of $\pm 2.5 \mathrm{~T}$, and 4 long solenoids with adiabatic field $2-5 \mathrm{~T}$. The 201.25 $\mathrm{MHz}$ linacs provide accelerating gradient $15 \mathrm{MV} / \mathrm{min}$ in the long straight sections. Four $1.3 \mathrm{~m}$ long liquid hydrogen absorbers are put between the linacs for transverse cooling, and $\mathrm{LiH}$ wedge absorbers in the short SS are used for the emittance exchange. To simplify the injection problem, $\pi-\mu$-production on an internal target is considered as an option for the MUCOOL experiment.
\end{abstract}

\section{INTRODUCTION}

The MUCOOL is an experimental program proposed for demonstration of ionization muon cooling and the hardware testing [1]. A possibility to use a ring cooler for the same goal is considered in this paper. The ring cooler has been proposed primarily as a relatively low cost facility for 3 -dimensional ionization cooling to produce a single lowemittance bunch for muon collider [2,3]. The scheme described below is based on a high frequency accelerating field and produces many bunches. The later is appropriate for a neutrino factory, but was not acceptable for a muon collider considered in earlier papers.

\section{DESCRIPTION OF THE COOLER}

Schematic layout of the ring cooler and its parameters are given in Fig. 1 and Table 1. The cooler consists of 8 dipoles providing a $45^{\circ}$ bend each, 4 short straight sections (SS), and 4 long SS. The dipoles have a non-zero gradient to provide focusing in both transverse directions. Solenoids are used to focus the beam transversely in the SS. The axial magnetic field alternates direction in each short SS, where solenoids of a relatively small radius are used to provide rather fast field flip. Lithium-hydride wedge absorbers are placed at a large vertical dispersion in the centers of the short SS. Direction of the field in the long SS is shown in Fig.1. The field grows from edges to center of the long SS to get a low $\beta$-function at the center where liquid hydrogen main absorbers are placed. Accelerating cavities are located inside the large-aperture solenoids.

\footnotetext{
* Work supported by the Universities Research Association, Inc., under contract DE-AC02-76CH03000 with the U. S. Department of Energy.

† balbekov@fnal.gov
}

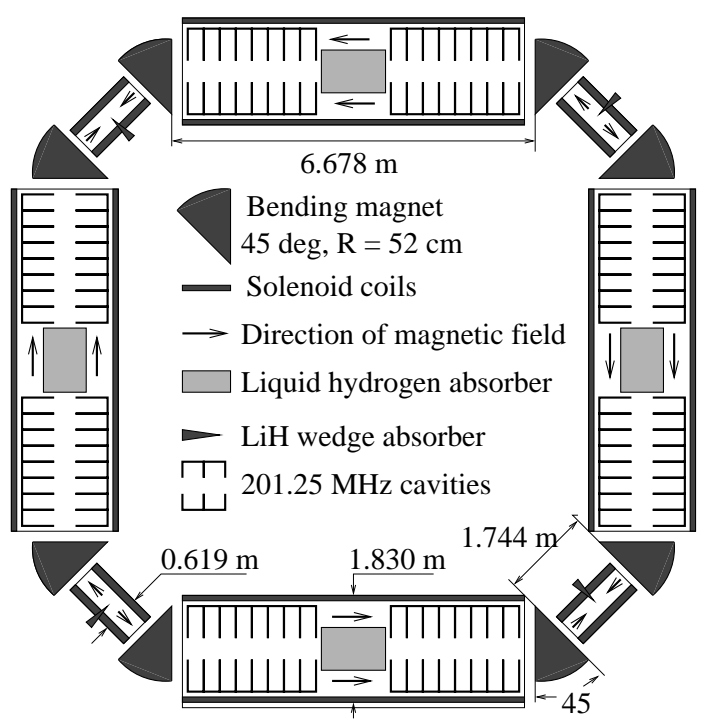

Figure 1: Layout of the ring cooler.

Table 1: Parameters of the ring cooler

\begin{tabular}{|l|l|}
\hline Circumference & $36.955 \mathrm{~m}$ \\
Nominal energy before/after absorber & $270 / 230 \mathrm{MeV}$ \\
Bending field & $1.453 \mathrm{~T}$ \\
Normalized field gradient & 0.5 \\
Maximal axial field of solenoid & $5.173 \mathrm{~T}$ \\
Revolution frequency & $7.1875 \mathrm{MHz}$ \\
RF harmonic number & 28 \\
Accelerating gradient & $15 \mathrm{MeV} / \mathrm{m}$ \\
$\mathrm{LH}_{2}$ main absorber, length & $133 \mathrm{~cm}$ \\
$\mathrm{LiH}$ wedge absorber, $d E / d y$ & $0.75 \mathrm{MeV} / \mathrm{cm}$ \\
\hline
\end{tabular}

\subsection{Bending Part and Short Straight Section}

Besides forming a quasi-circular beam orbit, the bending part should provide a dispersion required for the emittance exchange. The long SS should be dispersion-free, otherwise a particle can find itself at a "wrong" orbit after strong acceleration, resulting in an excitation of betatron oscillations. The dispersion in the "arcs" can be produced in a number of ways using bending magnets of different curvature or bent solenoids [2-3]. In this instance, we use a combination of 2 bending magnets and 2 solenoids with opposite directions of the field. Layout of the SS and its axial magnetic field are shown if Figs. 2 and 3. Note that the bending magnets are considered as perfect magnetic mirrors in calculation of the solenoid field. Transverse field of the bending magnets is introduced analytically including nonlinear part to satisfy Maxwell equations.

Dispersion functions $D_{x, y}$ of this part is shown in Fig.3. The $D_{x}$ is the same in all short SS, while the $D_{y}$ changes the sign in each section. Vertical wedge absorbers placed at 


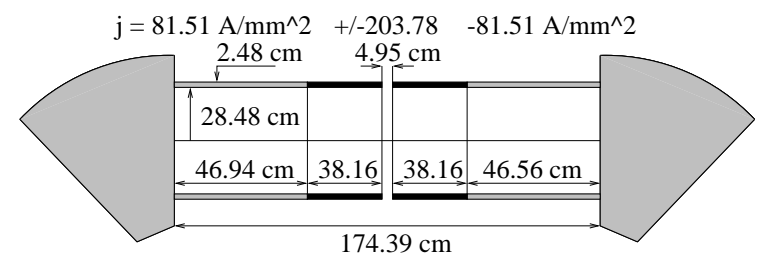

Figure 2: Layout of the short straight section.

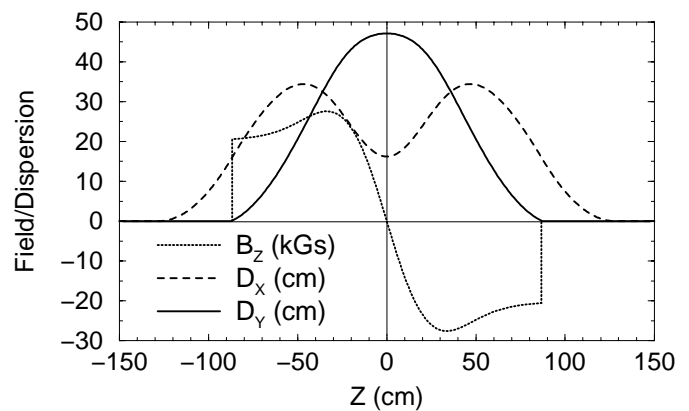

Figure 3: Axial field and dispersion functions in short SS.

the centers of the sections are used for emittance exchange. Each absorber cuts off only a half of the aperture to avoid energy loss of equilibrium particles. Material of the wedge absorbers is $\mathrm{LiH}$, and their gradient is $0.75 \mathrm{MeV} / \mathrm{cm}$ corresponding to the wedge angle of $25.4^{\circ}$.

\subsection{Long Straight Section}

Layout and axial magnetic field of the long SS are shown in Fig. 4. The coils containing 1 or 3 layers of conductor produce the axial field of 2.057 to $5.173 \mathrm{~T}$. The field at the edges provides the same transverse focusing as the bending magnets, that is correct matching of these parts. The growth of the field to the center allows to decrease a $\beta$-function at the absorbers approximately to $30 \mathrm{~cm}$ at the equilibrium energy. The liquid hydrogen absorbers occupy the region $\pm 66.7 \mathrm{~cm}$. There are two linacs per section $260 \mathrm{~cm}$ long each. The ring with such long SS is not resonance-free, and there are narrow stop bands spacing by energy intervals of 30-40 MeV. In practice 2 or 3 of them can be suppressed by a special choice of the coil parameters; however it is not always expediently because of dispersion. Actually, the long SS are dispersion-free only in a linear approximation in $\Delta p / p$. The nonlinear additions are non-zero in the long SS and can be quite significant near the resonances.

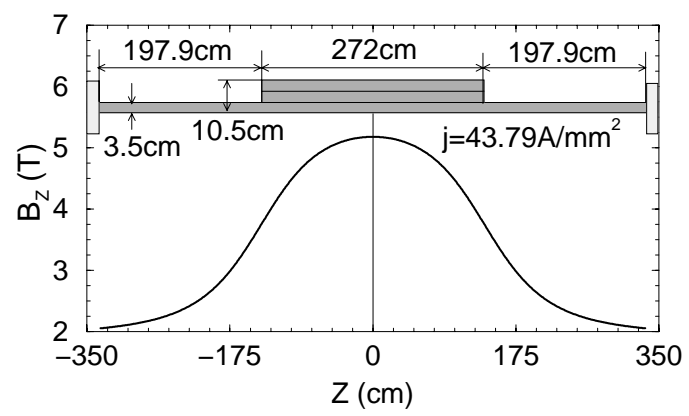

Figure 4: Layout and axial field in the long SS.
Therefore, the proposed scheme represents some compromise providing a maximum cooling rate and transmission by a partial suppression of the resonances and nonlinear dispersion. Some peculiarities of the longitudinal motion also were taken into account in the choice of the solenoid length: short solenoids would lead to decrease of the separatrix area and longitudinal acceptance, an excessive length leads to strong synchrotron resonances due to a high synchrotron frequency. It was found empirically that the optimal length of the long SS is 6 to $7 \mathrm{~m}$.

\section{SIMULATION}

\subsection{Cooling of Gaussian Beam}

Cooling of a Gaussian beam was studied to estimate the cooler performance. Initial parameters of the beam w.r.t. the center of the long SS are given in the first column of Table 2 . One should note that the energy spread of $18 \mathrm{MeV}$ was used originally, and energy-transverse momentum correlation was applied after that resulting in the total particle energy of

$E=E_{\text {ref }} \sqrt{1+\left(p_{t}^{2} / m^{2} c^{2}+r^{2} / \beta^{2}\right)_{\text {random }}}+\Delta E_{\text {random }}$,

where $E_{\text {ref }}=250 \mathrm{MeV}$ and $\beta=30 \mathrm{~cm}$ are the reference energy and transverse $\beta$-function at the center of the SS. The correlation is applied to take into account the dependence of the revolution frequency on the transverse momentum. Actually, it should arise at the bunching of the beam at the ring. Just this energy spread is shown in the Table 2.

Evolution of the beam parameters in the cooling process is presented in Fig. 5. Transverse cooling looks as expected; however considerable increase of the longitudinal emittance is observed during 5-6 turns after the beginning ( 1 turn $=4$ periods). Actually, it is an effect of a non-Gaussian tail containing the particles out the separatrix which should be lost soon. Mentioned above dependence of the revolution frequency on transverse momentum underlies this effect. The initial $\left(E-p_{t}\right)$-correlation mitigates it on average, but cannot prevent oscillations of the transverse momentum and revolution frequency which provides

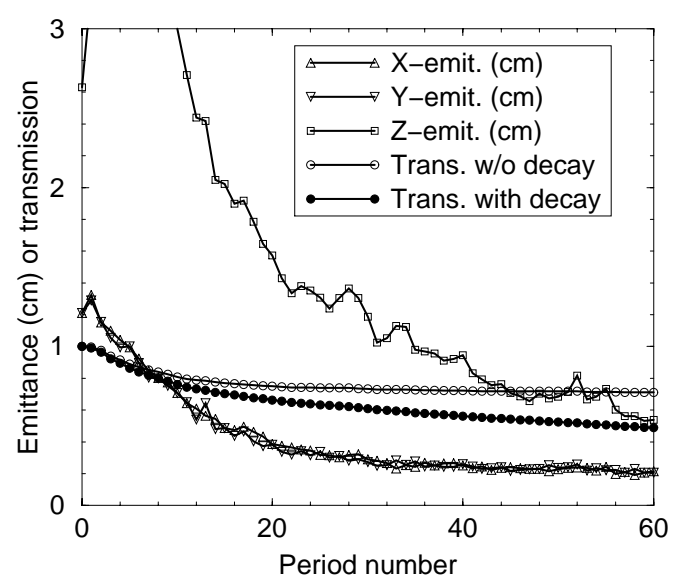

Figure 5: Beam emittance and transmission at the cooling. 
Table 2: R.m.s. parameters of the beam vs number of turns

\begin{tabular}{|l|r|r|r|}
\hline Number of turns & $\mathbf{0}$ & $\mathbf{1 0}$ & $\mathbf{1 5}$ \\
\hline Horizontal size $(\mathrm{mm})$ & 39.5 & 16.9 & 16.4 \\
Vertical size (mm) & 40.2 & 17.8 & 15.9 \\
Longitudinal size (mm) & 88.9 & 65.4 & 51.2 \\
Horiz. momentum $(\mathrm{MeV} / \mathrm{c})$ & 32.4 & 14.6 & 13.8 \\
Vertical momentum (MeV/c) & 31.9 & 14.5 & 13.8 \\
Energy spread (MeV) & 31.3 & 13.4 & 11.1 \\
Horizontal emittance (mm) & 12.1 & 2.34 & 2.15 \\
Vertical emittance (mm) & 12.1 & 2.44 & 2.07 \\
Longitudinal emittance (mm) & 26.3 & 8.31 & 5.38 \\
3D-emittance (mm $\left.{ }^{3}\right)$ & 3856 & 47.4 & 24.0 \\
Transmission w/o decay (\%) & 100 & 72.2 & 71.2 \\
Transmission with decay (\%) & 100 & 56.3 & 48.9 \\
\hline
\end{tabular}

significant perturbation of the synchrotron motion. In spite of this, during $10-15$ revolutions, the longitudinal emittance decreases by a factor of 3 to 4 and the 3D phase volume - by a factor of 100 to 150 with transmission of 50 to $60 \%$ (see Table 2).

\subsection{The Cooler with Internal Target}

One of the most serious problems is injection in the ring cooler. Usual way would require applying of a very strong large-aperture fast kicker magnet. Therefore, nontraditional solutions should be considered also. An interesting scheme with fast acceleration/deceleration to inject muons into the ring without kicker was proposed recently in Ref. [4]. A simpler scheme is considered below specifically for the MUCOOL experiment.

A proton beam is directed onto a target placed just in the beginning of a long SS of the ring. Pions generated in the target have a good chance to survive in the SS with a liquid hydrogen target. About $20 \%$ of the pions decay generating muons - candidates for the cooling. The key question is a long-term surviving of these muons in the ring with an internal target. First simulation was performed for a $15-\mathrm{cm}$ long black hole target, motivated by intensive muon scattering in any high- $Z$ material. The results - almost insensitive to the target length - are presented in Table 3 for several target radii. It seems that a $2.5 \mathrm{~mm}$ target looks quite acceptable for cooling demonstration.

These results are confirmed by full Monte Carlo simulations with the MARS code [5]. It turns out that for a $8 \mathrm{GeV}$ Fermilab Booster proton beam with $5 \times 10^{12} \mathrm{ppp}$, the copper target radius $2.5 \mathrm{~mm}$ is optimal both from pion production and target survival standpoints. Fig. 6 shows temperature rise distribution in a $15-\mathrm{cm}$ long copper target after such a

Table 3: Beam parameters after 15 turns vs the target radius

\begin{tabular}{|l|r|r|r|}
\hline Target radius (mm) & $\mathbf{2 . 5}$ & $\mathbf{5}$ & $\mathbf{7 . 5}$ \\
\hline Horizontal emittance (mm) & 2.04 & 2.03 & 2.11 \\
Vertical emittance (mm) & 2.12 & 2.08 & 2.30 \\
Longitudinal emittance (mm) & 5.47 & 5.87 & 6.58 \\
Transmission w/o decay (\%) & 58.6 & 42.8 & 30.0 \\
Transmission with decay (\%) & 40.2 & 29.4 & 20.6 \\
\hline
\end{tabular}

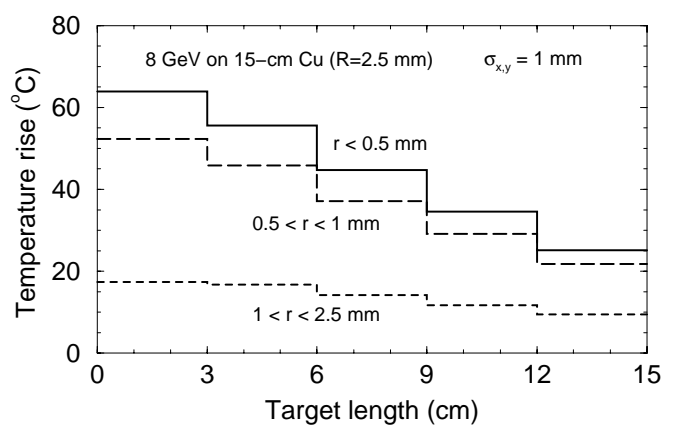

Figure 6: Instantaneous temperature rise in $2.5 \mathrm{~mm}$ radius copper target irradiated by a $8 \mathrm{GeV}$ beam of $5 \times 10^{12} \mathrm{ppp}$.

pulse. The peak $\Delta T \approx 70^{\circ} \mathrm{C}$, the peak energy deposition is $24.8 \mathrm{~J} / \mathrm{g}$, and total energy deposited in the target is $183 \mathrm{~J}$. All this is rather encouraging and now requires engineering consideration of a target cooling scheme.

About 0.015 pions of each sign per proton are generated in this target in the interval $p_{t}<100 \mathrm{MeV} / \mathrm{c}, 280<E<$ $420 \mathrm{MeV}$, approximately corresponding to the cooler acceptance. This gives $\sim 7.5 \times 10^{10}$ pions with $\sim 1 \times 10^{10}$ muons at the liquid hydrogen absorber. Pions surviving after the absorber would generate additionally $\sim 0.5 \times 10^{10} a$ muons in the downstream part of the straight section. Approximately a half of all muons falls in the ring cooler acceptance, and taking into account a 30\% RF capturing efficiency, the total number of muons in 28 bunches of the cooler is expected to be $2.5 \times 10^{9}$, i.e. $\sim 10^{8}$ muons per bunch before and 2.5 times less after the cooling.

\section{CONCLUSION}

It is shown that the high frequency ring cooler is capable to produce 3D muon cooling with a cooling factor of 100150 and transmission of $50-60 \%$, that is quite suitable for a neutrino factory. Injection/extraction in the ring requires additional studies. Pion/muon production in an internal target is considered also as an option for the MUCOOL experiment. The achievable beam emittance and cooling factor are approximately the same in this case, but the transmission falls to $30-40 \%$.

\section{REFERENCES}

[1] MUCOOL Proposal: Ionization Cooling R\&D Program for a High Luminosity Muon Collider, Fermilab proposal 204.

[2] V.I.Balbekov and A.Van Ginneken, "Ring Cooler for Muon Collider". In the book "Physics Potential and Development of $\mu^{=} \mu^{-}$Colliders". AIP Conf. Proc. 441, p.309 (1997).

[3] V.Balbekov, "Possibility of Using a Ring Accelerator for Ionization Cooling of Muons". PAC1999, V.1, p.315.

[4] D.Neuffer, "Injection and/or Extraction and a Ring Cooler". Fremilab Mucool Note 211, June 2001.

[5] N. V. Mokhov, "The MARS Code System User's Guide", Fermilab-FN-628 (1995); N. V. Mokhov and O. E. Krivosheev, "MARS Code Status", Fermilab-Conf00/181 (2000). http://www-ap.fnal.gov/MARS/. 